Please do not remove this page

RMIT

UNIVERSITY

\title{
Eigenvector methods for analysis of Human PPG, ECG and EEG signals
}

Übeyli, Elif Derya; Cvetkovic, Dean; Cosic, Irena

https://researchrepository.rmit.edu.au/esploro/outputs/9921863479501341/filesAndLinks?institution=61RMIT_INST\&index=null

Übeyli, E. D., Cvetkovic, D., \& Cosic, I. (2007). Eigenvector methods for analysis of Human PPG, ECG and EEG signals. Proceedings of the 29th Annual International Conference of the IEEE EMBS, Lyon, France. https://doi.org/10.1109/IEMBS.2007.4353036

Published Version: https://doi.org/10.1109/IEMBS.2007.4353036

Repository homepage: https://researchrepository.rmit.edu.au

(C2007 IEEE. Personal use of this material is permitted. However, permission to reprint/republish this material for advertising or promotional purposes or for creating new collective works for resale or redistribution to servers or lists, or to reuse any copyrighted component of this work in other works must be obtained from the IEEE.

Downloaded On 2023/04/27 01:24:17 +1000 


\title{
Eigenvector Methods for Analysis of Human PPG, ECG and EEG Signals
}

\author{
Elif Derya Übeyli, Dean Cvetkovic, Irena Cosic
}

\begin{abstract}
This paper presents eigenvector methods for analysis of the photoplethysmogram (PPG), electrocardiogram (ECG), electroencephalogram (EEG) signals recorded in order to examine the effects of pulsed electromagnetic field (PEMF) at extremely low frequency (ELF) upon the human electrophysiological signal behavior. The features representing the PPG, ECG, EEG signals were obtained by using the eigenvector methods. In addition to this, the problem of selecting relevant features among the features available for the purpose of discrimination of the signals was dealt with. Some conclusions were drawn concerning the efficiency of the eigenvector methods as a feature extraction method used for representing the signals under study.

Key Words: PPG, ECG, EEG, Eigenvector methods
\end{abstract}

\section{INTRODUCTION}

$\mathrm{T}$ HE entire process of methodologies developed for automated diagnosis can generally be subdivided into a number of disjoint processing modules: preprocessing, feature extraction/selection, and classification. Signal/image acquisition, artefact removing, averaging, thresholding, signal/image enhancement and edge detection are the main operations in the course of preprocessing. The accuracy of signal/image acquisition is of great importance since it contributes significantly to the overall classification result. The markers are subsequently processed by the feature extraction module. The module of feature selection is an optional stage, whereby the feature vector is reduced in size including only, from the classification viewpoint, what may be considered as the most relevant features required for discrimination. The classification module is the final stage in automated diagnosis. It examines the input feature vector and based on its algorithmic nature, produces a suggestive hypothesis [1].

Feature extraction is the determination of a feature or a feature vector from a pattern vector. For pattern processing problems to be tractable requires the conversion of patterns to features, which are condensed representations of patterns, ideally containing only salient information. Feature extraction methods are subdivided into: 1) statistical characteristics and 2) syntactic descriptions. Feature

Elif Derya Übeyli is with the TOBB Economics and Technology University, Faculty of Engineering, Department of Electrical and Electronics Engineering, 06530 Sögütözü, Ankara, Turkey (corresponding author phone: +903122924080 ; fax: +903122924091; e-mail: edubeyli@etu.edu.tr ).

Dean Cvetkovic is with the RMIT University, School of Electrical and Computer Engineering, GPO Box 2476V Melbourne VIC 3001, Australia (e-mail: dean.cvetkovic@rmit.edu.au).

Irena Cosic is with the RMIT University, School of Electrical and Computer Engineering, GPO Box 2476V Melbourne VIC 3001, Australia (e-mail: irena.cosic@,rmit.edu.au). selection provides a means for choosing the features which are best for classification, based on various criteria. The feature selection process performed on a set of predetermined features. Features are selected based on either 1) best representation of a given class of signals, or 2) best distinction between classes. Therefore, feature extraction/selection plays an important role in classifying systems such as neural networks. From the viewpoint of managing large quantities of data, it would still be most useful if irrelevant or redundant attributes could be segregated from relevant and important ones, although the exact governing rules may not be known. In this case, the process of extracting useful information from a large data set can be greatly facilitated [1]. In the present study, spectral analysis of the the photoplethysmogram (PPG), electrocardiogram (ECG), electroencephalogram (EEG) signals was performed by using eigenvector methods.

In the past few decades, the responses of human and animal EEG activity to non-ionising radiation of Extremely Low Frequency (ELF) have been studied [2]-[5]. Since that time, various studies have reported that humans and animals are particularly sensitive to ELF or ELF modulated sensory stimulation. The ELF refers to the range of electromagnetic field frequencies below $300 \mathrm{~Hz}$.

Several studies have examined the effects of sinusoidal ELF magnetic fields upon the human EEG activity in the past [2]. Cvetkovic and Cosic [3] single-blind counterbalanced pilot study investigated whether the human EEG activity could be altered when stimulated by localised ELF magnetic field at the top-central human head region. The statistical results performed on the recorded EEG data did reveal a significant difference between exposure and control, found in the Alphal EEG band $(7.5-9.5 \mathrm{~Hz})$ at the vertex head position, where magnetic field stimulation was applied at the Alpha1 frequency of $8.33 \mathrm{~Hz}$. It has been assumed that the effect in Alpha1 EEG findings is possibly related to 'synchronisation', 'induced rhythmic' and 'synchrony spread' theories of neuron firing rate after ELF magnetic field. Tabor et al. [4] study on 15 subjects revealed that the changes of time-domain Heart Rate Variability (HRV) parameters could be associated with the influence of $50 \mathrm{~Hz}$ magnetic field (20-30 $\mu \mathrm{T})$. Tabor's time-domain HRV parameters included linear and non-linear analysis. Baldi et al. [5] study on the influence of ELF PEMF exposure on the HRV using linear anlaysis, revealed a HR variation in all subjects.

In comparison to previous studies, the pilot study described in this paper consists of measuring the PPG, ECG, and EEG signal responses to ELF PEMF exposures over a 5 day period. A significant contribution of the present work 
was the computation of power levels of the power spectral density (PSD) estimations obtained by the eigenvector methods which were used to extract the representative features of the signals under study in order to obtain the accurate classification models.

\section{DATA DESCRIPTION}

The pilot experiment consisted of 1 healthy subject, recruited to participate for 5 consecutive days (excluding weekends). The experiment was a double-blinding and counter-balanced experimental design, where neither the subject nor the investigator was aware of the EMF exposure applied to the subject. At each sessions (day) the experimental protocol was designed to record the biosignals before (baseline) and after (post) EMF exposure during: control (magnetic field turned OFF) and exposure (magnetic field turned $\mathrm{ON}$ ). The experiment was conducted between 1 and $3 \mathrm{pm}$ for the 5 days. The ELF PEMF stimulations were undertaken with MEDEC Bioresonance Therapy System. The 10 minute ELF PEMF exposure were generated by the magnetic flux density at the top $(2.33 \mu \mathrm{T})$, middle $(5.24 \mu \mathrm{T})$ and bottom $(6.45 \mu \mathrm{T})$ of the applicator mattress at the operating frequency of $16 \mathrm{~Hz}$.

All the biosignals were recorded for 60 seconds after the ELF PEMF exposure. The subject was connected to MINDSET EEG machine to record the EEG activity of the brain using standard Neuroscan 19-electrode EEG cap. The cap was placed on subject's head according to $10 / 20$ International System. The Referential Montage of 16 channels was used throughout this investigation. The left brain hemisphere electrodes: Fp1, F7, F3, T7, C3, P7, P3 and $\mathrm{O} 1$ were all referenced to $\mathrm{M} 1$ or $\mathrm{A} 1$ (left masterioid). While the right brain hemisphere electrodes: Fp2, F8, F4, $\mathrm{T} 8, \mathrm{C} 4, \mathrm{P} 8, \mathrm{P} 4$ and $\mathrm{O} 2$ were referenced to right masteroid (M2 or A2). The EEG signals were sampled at the rate of $256 \mathrm{~Hz}$. Two other signals were recorded using Data Acquisition device BIOPAC Inc., which consisted of MP100A system with ECG100C Electrocardiogram amplifier (ECG) and PPG100C Photo-plethysmogram amplifier (PPG). The $50 \mathrm{~Hz}$ notch filter was activated to shield the subject and electrodes from the electric fields in the laboratory. The signals were digitised at a rate of $100 \mathrm{~Hz}$ and transmitted to a PC's Acqknowledge 3.7 software via USB cable. Recording took place in a dim room, inside a Faraday cage constructed of mesh wire and steel frames. Inside the cage, subjects were laid in a comfortable semi-reclining METRON chair.

\section{EIGENVECTOR METHODS}

Eigenvector methods are used for estimating frequencies and powers of signals from noise-corrupted measurements. The Pisarenko, multiple signal classification (MUSIC), and Minimum-Norm are the three eigenvector methods [6],[7]. The Pisarenko method is particularly useful for estimating PSD which contains sharp peaks at the expected frequencies. The polynomial $A(f)$ which contains zeros on the unit circle can then be used to estimate the PSD.

$$
A(f)=\sum_{k=0}^{m} a_{k} e^{-j 2 \pi f k}
$$

where $A(f)$ represents the desired polynomial, $a_{k}$ represents coefficients of the desired polynomial, and $m$ represents the order of the eigenfilter, $A(f)$. From the eigenvector corresponding to the minimum eigenvalue, the Pisarenko method determines the signal PSD from the desired polynomial:

$$
P_{\text {PISARENKO }}(f)=\frac{1}{|A(f)|^{2}} \text {. }
$$

The MUSIC method is also a noise subspace frequency estimator and eliminates the effects of spurious zeros by using the averaged spectra of all of the eigenvectors corresponding to the noise subspace. The resultant PSD is determined from

$$
P_{\text {MUSIC }}(f)=\frac{1}{\frac{1}{K} \sum_{i=0}^{K-1}\left|A_{i}(f)\right|^{2}}
$$

where $K$ represents the dimension of noise subspace, $A_{i}(f)$ represents the desired polynomial that corresponds to all the eigenvectors of the noise subspace.

In addition to the Pisarenko and MUSIC methods, the Minimum-Norm method was investigated. In order to differentiate spurious zeros from real zeros, the MinimumNorm method forces spurious zeros inside the unit circle and calculates a desired noise subspace vector $a$ from either the noise or signal subspace eigenvectors. Thus, while the Pisarenko method uses only the noise subspace eigenvector corresponding to the minimum eigenvalue, the MinimumNorm method uses a linear combination of all noise subspace eigenvectors. The Minimum-Norm PSD can be estimated as follows:

$$
P_{\text {MIN }}(f, K)=\frac{1}{|A(f)|^{2}}
$$

where $K$ represents the dimension of the noise subspace [6],[7].

\section{RESULTS AND DISCUSSION}

The eigenvector methods are based on an eigendecomposition of the correlation matrix of the noisecorrupted signal. Even when the signal-to-noise ratio (SNR) is low, the eigenvector method produces a frequency spectrum of high resolution. These methods provide sufficient resolution to estimate the sinusoids from the data. Hence, to gain some noise immunity it is reasonable to retain only the principal eigenvector components in the estimation of the autocorrelation matrix. The Pisarenko, MUSIC, and Minimum-Norm methods were employed to obtain the PSDs of the PPG, ECG, and EEG signals. Using the frequency estimations provided by any one of these methods, the power levels of the signal can be determined from the power matrix. In the Pisarenko method, the eigenvector associated with the minimum eigenvalue of the estimated 
autocorrelation matrix is used to calculate the PSD. This method may produce spurious zeros and has a relatively poor statistical accuracy. In all cases, the Pisarenko PSD showed extra peaks as compared to the PSDs obtained from the MUSIC or Minimum-Norm methods (Fig. 1-3). Since the Pisarenko method showed tendency to generate spurious zeros, the Pisarenko was considered inappropriate for spectral analysis of the PPG, ECG, EEG signals. The MUSIC method eliminates these spurious zeros by averaging the spectra from all of the eigenvectors corresponding to noise subspace. The MUSIC method is the most widely studied, computationally simple, highresolution eigenvector method. From Fig. 1-3, it is apparent that the MUSIC method can be considered as an appropriate method for spectral analysis of the PPG, ECG, EEG signals. The Minimum-Norm method treats the problem of spurious zeros by forcing them inside the unit circle (Fig. 1-3). Both the MUSIC and Minimum-Norm methods produce similar spectral characteristics with nearly identical peak frequencies. This similarity held for all the signals under study indicating that the power levels can be accurately estimated using either method. For each signal the 129 points of the logarithm of the power levels of the PSDs were computed by the three eigenvector methods.

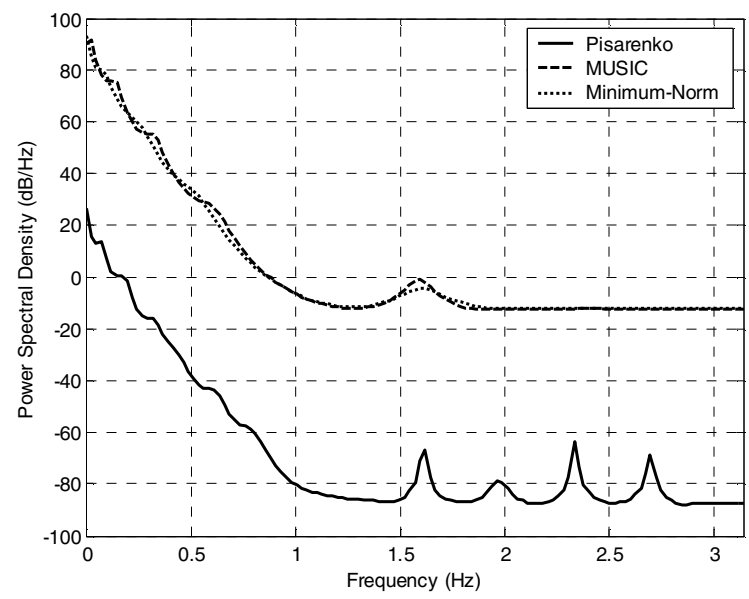

Fig. 1. PSDs of PPG obtained by Pisarenko, MUSIC, Minimum-Norm

Feature selection is an important component of designing the neural network based on pattern classification since even the best classifier will perform poorly if the features used as inputs are not selected well. The power levels of the PSDs of the PPG, ECG, EEG signals of each record were used as the feature vectors representing the signals. In order to reduce the dimensionality of the extracted feature vectors, statistics over the set of the power levels of the PSDs obtained by the three eigenvector methods was used. The following statistical features were used to represent the time-frequency distribution of the signals under study:

1. Maximum of the power levels of the PSDs in each signal segment.

2. Mean of the power levels of the PSDs in each signal segment.
3. Minimum of the power levels of the PSDs in each signal segment.

4. Standard deviation of the power levels of the PSDs in each signal segment.

Tables I and II present the extracted features of exemplary PPG, ECG, EEG records. From Tables I and II, one can see that the extracted features of the different PPG, ECG, EEG signals are different from each other. Therefore, we decided that they can serve as useful parameters in representing the PPG, ECG, EEG signals.

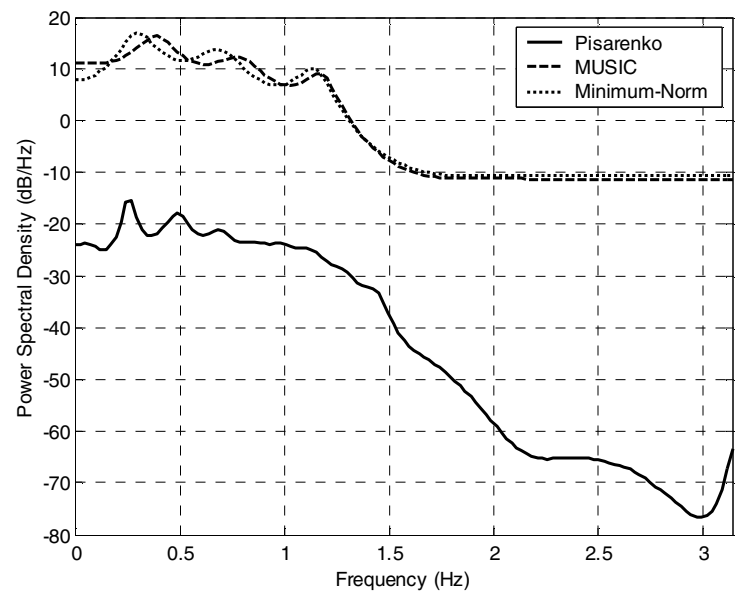

Fig. 2. PSDs of ECG obtained by Pisarenko, MUSIC, Minimum-Norm

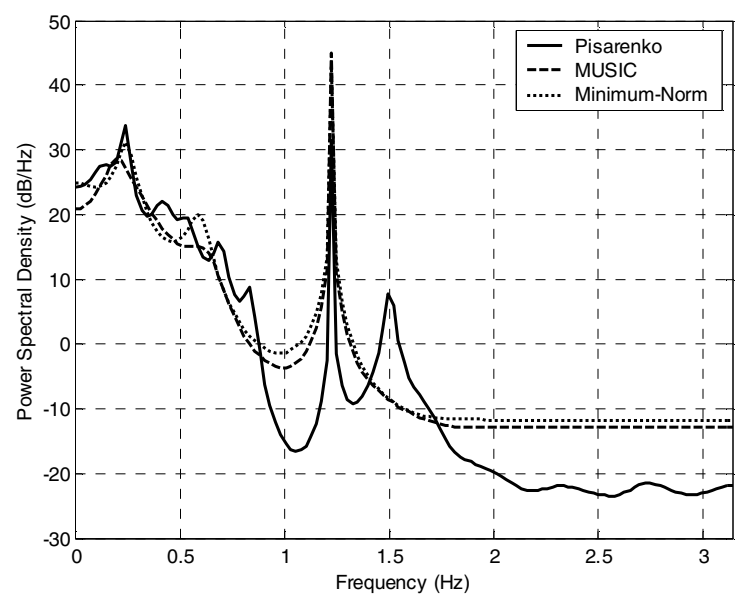

Fig. 3. PSDs of EEG obtained by Pisarenko, MUSIC, Minimum-Norm

\section{CONCLUSION}

Since classification is more accurate when the pattern is simplified through representation by important features, feature extraction and selection play an important role in classifying systems such as neural networks. In the present study, feature extraction from the PPG, ECG, EEG signals was performed by usage of the eigenvector methods. The study demonstrated that the power levels of the PSDs obtained by the eigenvector methods can be used as the representing features of the PPG, ECG, EEG signals. 
TABLE I

EXTRACTED FEATURES OF EXEMPLARY PPG AND ECG RECORDINGS

\begin{tabular}{|c|c|c|c|c|}
\hline Dataset & Extracted Features & $\begin{array}{c}\text { Pisarenko PSD } \\
\text { values }\end{array}$ & $\begin{array}{l}\text { MUSIC PSD } \\
\text { values }\end{array}$ & $\begin{array}{l}\text { Minimum-Norm } \\
\text { PSD values }\end{array}$ \\
\hline \multirow{4}{*}{$\begin{array}{c}\text { PPG } \\
\text { (record 1) }\end{array}$} & Maximum & 26.4057 & 91.6023 & 93.5343 \\
\hline & Mean & -67.6325 & 4.2297 & 4.0555 \\
\hline & Minimum & -87.7745 & -12.7587 & -12.2335 \\
\hline & Standard deviation & 29.3020 & 27.8214 & 27.2225 \\
\hline \multirow{4}{*}{$\begin{array}{c}\text { ECG } \\
\text { (record 1) }\end{array}$} & Maximum & -15.4566 & 16.2387 & 16.8676 \\
\hline & Mean & -43.9118 & -1.6080 & -1.3450 \\
\hline & Minimum & -76.6836 & -11.3676 & -10.7013 \\
\hline & Standard deviation & 20.5890 & 10.7358 & 10.3397 \\
\hline \multirow{4}{*}{$\begin{array}{c}\text { PPG } \\
\text { (record 2) }\end{array}$} & Maximum & 39.5926 & 96.8626 & 95.8678 \\
\hline & Mean & -69.9416 & 1.6860 & 1.8838 \\
\hline & Minimum & -90.2691 & -14.5815 & -14.2972 \\
\hline & Standard deviation & 29.2255 & 27.1035 & 26.9743 \\
\hline \multirow{4}{*}{$\begin{array}{c}\text { ECG } \\
\text { (record 2) }\end{array}$} & Maximum & -17.6046 & 18.4919 & 17.2507 \\
\hline & Mean & -46.3434 & -3.3588 & -3.1877 \\
\hline & Minimum & -83.6701 & -14.7214 & -14.4158 \\
\hline & Standard deviation & 21.3649 & 12.2141 & 12.0930 \\
\hline
\end{tabular}

TABLE II

EXTRACTED FEATURES OF EXEMPLARY EEG RECORDINGS

\begin{tabular}{|c|c|c|c|c|}
\hline Dataset & Extracted Features & $\begin{array}{c}\text { Pisarenko PSD } \\
\text { values }\end{array}$ & $\begin{array}{c}\text { MUSIC PSD } \\
\text { values }\end{array}$ & $\begin{array}{l}\text { Minimum-Norm } \\
\text { PSD values }\end{array}$ \\
\hline \multirow{4}{*}{$\begin{array}{c}\text { Record 1 } \\
\text { (channel 1) }\end{array}$} & Maximum & 33.6884 & 45.0685 & 42.7587 \\
\hline & Mean & -5.9108 & -1.7742 & -0.6818 \\
\hline & Minimum & -23.4977 & -12.9111 & -11.8994 \\
\hline & Standard deviation & 18.1492 & 14.1142 & 14.1525 \\
\hline \multirow{4}{*}{$\begin{array}{c}\text { Record } 1 \\
\text { (channel 2) }\end{array}$} & Maximum & 33.5918 & 43.4839 & 46.0228 \\
\hline & Mean & -8.8042 & -0.0179 & 0.7233 \\
\hline & Minimum & -28.0775 & -12.8849 & -11.9000 \\
\hline & Standard deviation & 20.2313 & 16.2059 & 15.7700 \\
\hline \multirow{4}{*}{$\begin{array}{c}\text { Record } 2 \\
\text { (channel 1) }\end{array}$} & Maximum & 37.0321 & 40.3150 & 39.8305 \\
\hline & Mean & -5.3407 & -0.6843 & -0.0320 \\
\hline & Minimum & -24.4418 & -12.8826 & -11.9080 \\
\hline & Standard deviation & 18.8253 & 14.7807 & 14.5577 \\
\hline \multirow{4}{*}{$\begin{array}{c}\text { Record } 2 \\
\text { (channel 2) }\end{array}$} & Maximum & 35.7484 & 47.1592 & 45.4446 \\
\hline & Mean & -8.0507 & 0.7355 & 0.8266 \\
\hline & Minimum & -28.9941 & -12.8531 & -11.9061 \\
\hline & Standard deviation & 20.9302 & 16.5884 & 15.7756 \\
\hline
\end{tabular}

[5] E. Baldi, C. Baldi, and B.J. Lithgow, "A Pilot Investigation of the Effets of Extremely Low Frequency Pulsed Electromagnetic Fields on Human's Heart Rate Variability," Bioelectromagnetics, vol. 28, pp. 64-68, 2007.

[1] N. Kwak, and C.-H. Choi, "Input feature selection for classification problems," IEEE Transactions on Neural Networks, vol. 13, no. 1, pp. 143-159, 2002.

[2] G.B. Bell, A.A. Marino, and A. Chesson, "Alterations in brain electrical activity caused by magnetic fields: detecting the detection process," Electroencephalogr Clin Neurophysiol, vol. 83, pp. 389-397, 1991.

[3] D. Cvetkovic, and I. Cosic, "Automated ELF Magnetic Field Stimulation of the Human EEG Activity," Integrated Computer-Aided Engineering, vol. 13, no. 4, pp. 313-328, 2006.

[4] Z. Tabor, J. Michalski and E. Rokita, "Influence of $50 \mathrm{~Hz}$ Magnetic Field on Human Heart Rate Variability: Linear and Nonlinear Analysis," Bioelectromagnetics, vol. 25, pp. 474-480, 2004.

[6] M. Akay, J.L. Semmlow, W. Welkowitz, M.D. Bauer, and J.B. Kostis, "Noninvasive detection of coronary stenoses before and after angioplasty using eigenvector methods," IEEE Transactions on Biomedical Engineering, vol. 37, no. 11, pp. 1095-1104, 1990.

[7] E.D. Übeyli, and İ. Güler, "Comparison of eigenvector methods with classical and model-based methods in analysis of internal carotid arterial Doppler signals," Computers in Biology and Medicine, vol. 33, no. 6, pp. 473-493, 2003. 